\title{
A TOOLKIT FOR DEVELOPING PROGRAMS OF CARD GAMES PLAYED ON THE INTERNET
}

\author{
Masato Koide and Jingde Cheng \\ Department of Information and Computer Sciences, Saitama University \\ koide@aise.ics.saitama-u.ac.jp and cheng@ics.saitama-u.ac.jp
}

\begin{abstract}
We propose some general-purpose protocols, which are necessary for playing card games on the Internet. The protocols govern the expressions of cards, the communication among players, servers, and other components within a card game played on the Internet. Based on the protocols, we have designed and implemented a toolkit for programming card games on the Internet. By using our toolkit, people can easily implement various card game programs without much programming skills or expert knowledge of computer science. We show some examples of how to develop card game programs using our toolkit.
\end{abstract}

Keywords: Internet, card game, protocols, and information security

\section{Introduction}

In recent years, it is becoming popular to play the Internet games. Various game servers have been developed and used [1] [2]. However, these game servers provide different protocols for not only communications on the Internet, but also representation of game rules. There are no general protocols which can cover two or more games. As a result, by the existence of protocols for communication, developing game programs is easy.

However, the development of game programs is still difficult for people who do not have programming skills or expert knowledge of computer science. In this case, tools for developing game programs are useful for the programming-laymen, and even for game programmers.

In [3] we have proposed some general-purpose protocols for card games played on the Internet. Our protocols govern communication among players, representation of game rules, and information security for playing card games on the Internet. Based on the protocols, we designed and implemented a toolkit for developing card game programs. By us-

The original version of this chapter was revised: The copyright line was incorrect. This has been corrected. The Erratum to this chapter is available at DOI: 10.1007/978-0-387-35660-0_65 
ing this toolkit anyone can easily implement game programs without much programming skills or expert knowledge of computer science.

\section{General-Purpose Protocols for Card Games Played on the Internet}

\subsection{Design Policy}

Card games performed on the Internet have many common actions. For example, actions of players of "discard" or "take cards from stock" are common in many card games, however, actions like "tear cards of" are not used generally. Below we pay attention to the common actions, and to the rules which specify whether those actions are effective. We classify and provide the set of actions as a library. It enables us to express the rule of a card game by choosing and combining the required rules from the library.

Moreover, an expression of cards, an expression of player's actions, and information security are needed for communication among players. So, we define them as protocols.

\subsection{Definition of "Card Games"}

We define "card games" as follows.

- Card games are played with playing cards.

- In all games two or more players shall exist.

- Games that requires player's reflex (such as "Seven Bridge" or "Speed") are not included because transmission speed based on each player's environment may become cause of an unfair game result.

- There is a "dealer" who is a referee and also a chairperson of games. The dealer shall know all information of a game, and shall supervise whether a player's action is according to the rules, and whether there is not any injustice.

- A person who actually participates in a game is called a "player", and a person who observes a game or waits for a game is called a "standby person". Players, standby persons and dealers are called "participants" in the game (see Fig. 1).

\subsection{Requirements to General-Purpose Protocols}

Below we list the requirements for general-purpose protocols when designing the Internet card games. They are: 
- Expression of cards

- Representation of actions of participants

- Description of rules

- System configuration

- Proceeding of games

- Information security

\subsection{Definition of protocols}

Expression of cards We define expression of cards based on "Contract Bridge" which is very popular card game. Each card is expressed with two characters. The first character expresses the suit of the card, and the next character expresses the rank of the card. For example, "2 of the heart" is expressed as "H2" and "Jack of the spade" is expressed as "SJ". Joker is expressed as "J0" or "J1". 0 or 1 is the rank of Joker. Such rank is used in some games in which Joker has ranking.

Representation of actions of participants We represent a participant's actions by one word (see Tab. 1). Some actions are in need of additional information. For example, the "Discard" command needs the number of discarding cards.

Table 1. Part of participant's actions

\begin{tabular}{cll}
\hline command & content & who can use \\
\hline DISCARD & discard cards from a hand & player \\
DRAW & draw cards from a stock or other player & player \\
DEAL & deal cards to players & dealer \\
ACTION & notify a player's action to other participants & dealer \\
LOGIN & become player & standby person \\
EXIT & exit game system & standby person \\
\hline
\end{tabular}

Description of rules To describe rules of games, we prepare a library named "Condition Set". This library includes some portions of rules of card games such as limit of the number of players, or in which case a certain action is effective .

System configuration Our general-purpose protocols assume that a game is played by the following system configurations (see Fig. 2). 
A game system is implemented based on server-client model. Client is considered as a player or a standby person, and Server is considered as a dealer. All clients connect to server, and any clients do not connect to the other clients. In addition, there is certificate authority (CA), and $\mathrm{CA}$ attests players.

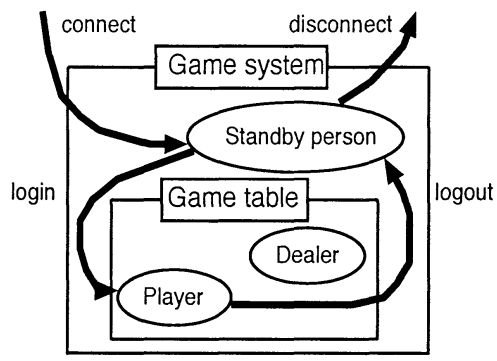

Figure 1. Library and protocols

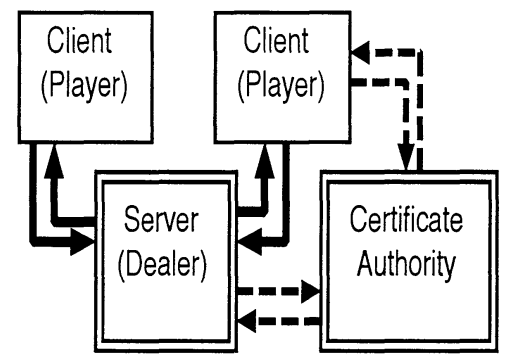

Figure 2. Change of user's state

Proceeding of games A game proceeds with the directions of a dealer. If the players are assembled, a dealer will proceed a game according the following five procedures.

1 A dealer notifies now whose turn it is to the participants.

2 A dealer notifies the list of actions which can be used for the player whose turn it is.

3 The player sends the action which he/she wants. If necessary, he/she adds some additional information.

$4 \mathrm{~A}$ dealer checks the action. If the action is against the rule of the game, a dealer asks the player to re-choose the action. If the action is not against the rule, a dealer notifies all participants of the action and the result, and returns to procedure 2 .

5 Until the end conditions of the game are fulfilled, the procedures from 1 to 4 are repeated.

A standby person can become a player if that happens before a game starts, and of course, in case the game is not a formal convention.

Information security The game program should have some information security functions, in order to (1) build a condition for players to 
trust each other, (2) to trust the game server and (3) to enjoy the game. A program must fulfill information with confidentiality and integrity; it must avoid spoofing or any other injustice. For that purpose, it is necessary to encrypt communication data, and to attest a player.

\subsection{Classification of General-Purpose Protocols}

Some protocols are applicable to card games as well as to various games played on the Internet. Changing the viewpoint, card games can be able to inherit a new Internet game. So, we distinguish the protocols by class. Some protocols that are applicable to various games are named "the Internet Game Protocol", other protocols that are applicable only to card games are named "Card Game Protocol", and protocols for information security are named "Information Security Protocols". Protocols for a new kind of game can be designed by inheriting the Internet Game Protocols (see Fig. 3).

\section{A Toolkit for Developing Card Game Programs}

\subsection{Requirements to a Toolkit}

Below follow six requirements to a toolkit for developing arbitrary programs of card games played on the Internet.

- Various card games can be implemented.

- Both server and client programs can be developed.

- Little programming skills and expert knowledge of computer science are needed for program development.

- The programs developed using a toolkit should operate on various platforms.

- The programs developed using a toolkit should have information security functions.

- A graphical User Interface is provided for players who enjoy the game very much.

To implement various card games, the design of our toolkit is based on our general-purpose protocols.

\subsection{Implementation of our Toolkit}

Our toolkit includes development tools for a server and a client program. The tools for client programs are for the development of a user 
interface for the players. Since a server carries out a dealer's duty, the tools for server programs treat the description of the rules of a game, and the management of the clients. These tools are classified into two categories based on the general-purpose protocols. One is applicable to games played on the Internet at large, the other is applicable to card games only.

We implemented our toolkit in Java. So, the implemented program using our toolkit operates platform-independently. Additionally, it can develop various card games since the design of our toolkit is based on the general-purpose protocols.

\subsection{Usage of our Toolkit}

Our toolkit provides fundamental functions, such as communication among players, or client management; a developer should make only rules of games him/herself. To describe the rules, a developer should choose and write down some necessary Condition Set. Our toolkit provides a Condition Set of about 60 conditions. When a rule cannot be described with the Condition Set, a developer can inherit or overwrite some tools. So, the quantity of programming can be lessened as much as possible.

The development of games using our toolkit can be compared to blocks play (see Fig. 4). The actual example of using a toolkit is shown in the next section.

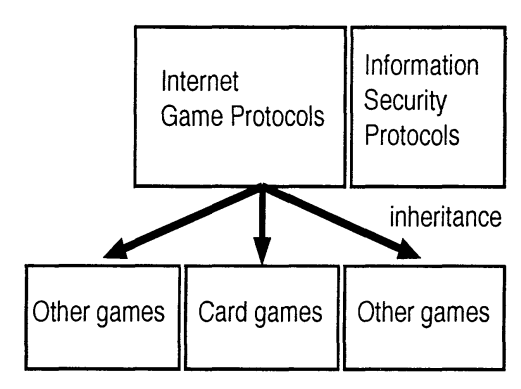

Figure 3. Division by class of general-purpose protocols

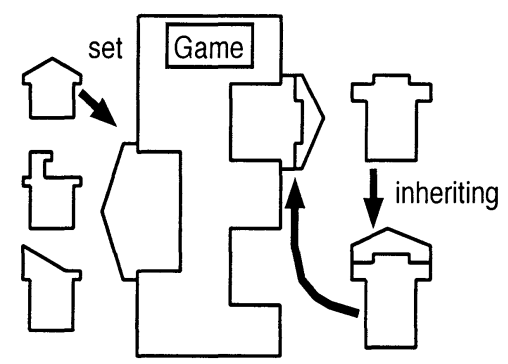

Figure 4. Developing games like blocks play 


\section{Experiments and Evaluation}

\subsection{Experiments}

To evaluate our general-purpose protocols and toolkit, we have implemented two card games using our toolkit. The games are "Page One" and "Hoist", of which the rules are unique for each of them. "Page one" is the game with which the player discards one card from the hand in order, and the player who discards the whole hand first wins. "Hoist" is a kind of game called a trick game; it is a game in which each player takes out one card from the hand at a time, competes for the superiority of that card, and determines victory or defeat by score. So, implementing these different games, we can evaluate the generality of our generalpurpose protocols. Moreover, we evaluate the usability of our toolkit by comparing it with the case of not using the toolkit.

\subsection{Experimental Results and Evaluation}

We were able to implement two games using our toolkit. For Hoist we described only one rule Condition Set currently prepared. However, for Page One we had to write a little program ourselves.

Although we described almost all rules with some Condition Set currently prepared, we had to write some source code to describe the rule "When the card of the same suit or rank as the card which the previous player took out is in a hand, the player cannot draw a card from a stock". This rule is peculiar of Page One. Fortunately, we were able to describe this rule by overwriting one rule out of the Condition Set. Moreover, we were able to lessen the quantity of programming. The source code is uploaded and can be seen at http://www.aise.ics.saitamau.ac.jp/ ${ }^{\sim}$ koide/cardgame.

Moreover, comparing with the case of not using our toolkit, the quantity of the source code decreased to about $8 \%$.

\section{Conclusions}

In this paper we proposed some general-purpose protocols for playing card games on the Internet. These protocols govern communication among players, expression of cards and player's actions, rules for game proceeding, and information security.

Based on the protocols we designed and implemented a toolkit for developing programs of card games played on the Internet. We implemented functions of network communications, expression of cards and player's actions, rules for game proceeding, data management, client management, and a character-based interface. 
To evaluate our protocols and toolkit, we developed two unique games using our toolkit. As a result, comparing them with the case of not using our toolkit, we implemented programs by choosing and writing down some necessary Condition Set. So, we may confirm that a developer can develop card game programs even if he/she has almost no programming skills and knowledge of computer science. Additionally, the quantity of a source code decreased to about $8 \%$. So, we can confirm the generality of our protocols and the usability of our toolkit.

We are implementing functions of information security, bidding, scoring, and GUI and going to add them to our toolkit. The goal of our toolkit is that anyone can develop game programs without programming skills and knowledge of computer science at all.

\section{References}

[1] Yahoo! Inc, "Yahoo! Games", http://games.yahoo.co.jp

[2] Blue Chip Bridge Ltd, "Blue Chip Bridge", http://www.bluechipbridge.co.uk/ protocol.htm

[3] M. Koide and J. Cheng, "General-purpose Protocols for Playing Card Games on the Internet", Proc. IPSJ 6th Game Programming Workshop (GPW'01), pp. 78-85, Hakone, Japan, October 2001 (in Japanese).

[4] H. Tabuchi, "International Security Standards Introduction of ISO/IEC 17799", Ohm-sha, 2000 (in Japanese).

[5] H. Tabuchi, "International Security Standards Introduction of ISO/IEC 15408 ", Ohm-sha, 2001 (in Japanese).

[6] M. Ruro and I. Durdanovic, "An Overview of NECI's Generic Game Server", Proc. IPSJ 6th Game Programming Workshop (GPW'01), pp. 72-77, Hakone, Japan, October 2001.

[7] B. Pell, "A Strategic Metagame Player for General Chess-like Games", Computational Intelligence 12(2), 177-198, 1996.

[8] B. Pell, "Metagame: A New Challenge for Games and Learning", Heuristic Programming in Artificial Intelligence 3 - The Third Computer Olimpiad, 1992.

[9] S. Shirou, "Card Games", Nitto-Shoinn, 1985 (in Japanese).

[10] American Contract Bridge League Home Page, http://www.acbl.org/

[11] Using the Eiffel Card Game Classes, http://www.cs.rit.edu/ icss232/ proj2/cardgame.html

[12] H.Komatsu, "PKI Hand Book", Soft reserch center (in Japanese).

[13] Netscape Communications Corporation, "Introduction to Public-Key Cryptography", http://developer.netscape.com/docs/manuals/security/pkin/ contents.htm 